\title{
Erratum
}

\section{Pineal Melatonin in a Sub-chronic Tryptophan Depletion Female Rat Model of Treatment-resistant Depression}

\section{Franklin¹, N. Hlavacova² ${ }^{2}$ S. Babic ${ }^{2}$, I. Bermudez ${ }^{1}$, D. Jezova ${ }^{2}$}

${ }^{1}$ School of Life Sciences, Oxford Brookes University, Oxford OX3 OBP, UK

${ }^{2}$ Institute of Experimental Endocrinology, Laboratory of Pharmacological Neuroendocrinology, Slovak Academy of Sciences, Bratislava, Slovakia

Pharmacopsychiatry 2015; 48

DOI $10.1055 / \mathrm{s}-0035-1554711$

Figure 1 was an uncorrected version. On Paragraph "TRP depletion was confirmed..." was a part of a sentence missing and the reference numbers are wrong.

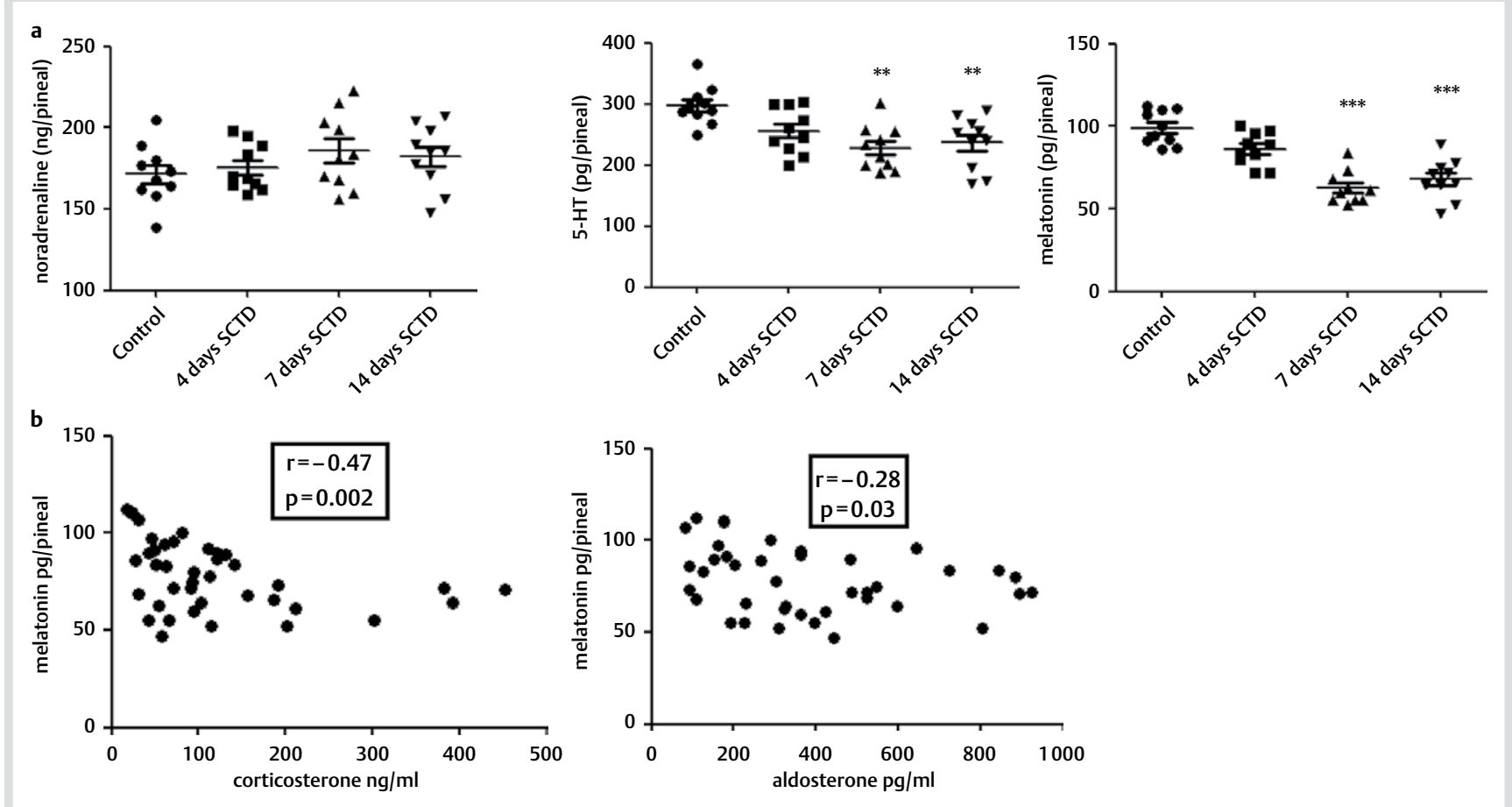

Fig. 1 Shows effects of TRP depletion over various periods of time on a pineal NA, 5-HT and melatonin respectively and $\mathbf{b}$ correlations over all groups $0-14$ days (Spearmon's) between pineal melatonin vs. serum corticosterone and aldosterone respectively. One-way Analysis of Variance for pineal NA ( $F=1.15$; $\mathrm{DF} 3,36 ; \mathrm{p}=0.34), 5-\mathrm{HT}(\mathrm{F}=6.91 ; \mathrm{DF}=3,36 ; \mathrm{p}<0.001)$ and melatonin $(\mathrm{F}=24.99 ; \mathrm{DF}=3,36 ; \mathrm{p}<0.0001)$ respectively.

TRP depletion was confirmed in these studies and was similar to that found in our previous studies [9] hence the model was validated. Both pineal melatonin and 5-HT content were significantly reduced in TRP depleted animals even as early as after 4 days of depletion. Interestingly, a very recent article demonstrates that 5-HT depletion in mice disrupts their sleep-wake cycle and motor activity [4]. It is therefore plausible that similar 5-HT depletion and subsequent pineal melatonin reductions in female rats here could indeed have the same end-point effects. Interestingly, patients with severe depression also demonstrate reduced melatonin levels in parallel with sleep disturbance [3]. Both serum corticosterone and aldosterone were increased in TRP depleted animals [11] and aldosterone has a causal relationship with the development of anxiety and depression-like behaviour [12] and is a common feature in patients with resistant depression [1]. Significant correlations between pineal melatonin and these hormones (even though that for aldosterone was less strong) underpin our assumption that sleep quality may be disrupted in TRP depleted animals. Pineal NA content was generally unaltered by the TRP depletion process and thus suggests that this played no part in other ongoing biochemical and behavioural changes at this time. 